\title{
METHOD OF HYSTERESIS CALCULATION IN A HYSTERESIS CONTROL CURRENT REGULATOR WITH CURRENT REFERENCE ADAPTATION
}

\author{
Stanislav Koschinsky, Dmitry Tey \\ Radioelectronics Department, State Technical University of Orel, 40, Naugorskoye Shosse, 302020 Orel, Russia \\ tel: 70862 419879, fax: 70862 416684, e-mail: tey@rambler.ru, kipra@ostu.ru
}

\begin{abstract}
In this paper hysteresis adaptation method is presented. It provides the algorithm to calculate hysteresis value equal to the maximum pulsation of current. This allows decrease disadvantage of hysteresis regulator with clocked commutation in application with variable reference.
\end{abstract}

Keywords: Hysteresis calculation algorithm, adaptive hysteresis control current regulator.

\section{REVIEW OF RELAY CURRENT REGULATORS AND MODERN TENDENCY OF RELAY CONTROL DEVELOPMENT}

Relay regulators have the best dynamic behavior among all pulse regulators. A high quick-action, simplicity are advantages of these regulators [1]. The operation principle of the simple pulse relay current regulators with one-stand relay border is shown in Fig. 1(a: switch is in conduction state by synchronization pulse, in non-conduction if current reaches the reference value and vice-versa for $b$ )).

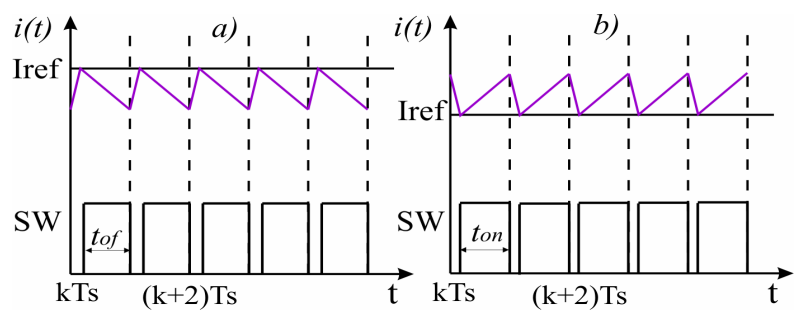

Fig. 1 - Time diagrams of relay current regulators with a) top-stand relay border b) low-stand relay border

Such kinds of the synchronized relay current regulators with one-stand relay border have evidential advantages and two important disadvantages. The principle instability in the whole range of a duty cycle variation is the first one (regulator with top-stand relay border and with lowstand relay border is unstable in the range of duty cycle from 0.5 to 1 and from 0.5 to 0 respectively). The second one is a static control error, which exists within the whole range of duty cycle variation.
The synchronized relay current regulator, which implements automatic change from the algorithm with top-stand relay border to the algorithm with low-stand relay border, was made in the 1975 [2]. But it needs the supplementary device, which detects a critical region $d=0.5$ and realizes the automatic change of regulation algorithms. Such mode of regulation was used in the high-speed electric train ER200 [1], which had exploited within the 19841995 on the railway line Moscow-St. Petersburg. At the end of 80-th some scientists almost simultaneously proposed the structure of two-stand relay current regulator that is synchronized with a double switching sequences - so-called the hysteresis controlled current regulator with clocked commutation (HCCRCC) [3,4]. It implements automatic changing from the algorithm with topstand relay border to the algorithm with low-stand relay border and guaranties the stability of synchronized periodic mode within the whole range of duty cycle variation without the supplementary device using. The time diagrams of HCCRCC are shown in Fig. 2. This regulator doesn't lose advantages of the relay current regulator with onestand relay border - the simplicity of implementation and the high quick-action, but it doesn't solve the static control error problem, which exists in the whole range of duty cycle variation.

Two main directions in the middle of 90-th were determined to minimize or practically exclude the static control error. The first one consists in a hysteresis adaptation [5], second one - in adaptation of a current reference [1]. In turns, the hysteresis adaptation method divides into a geometrical 
method $[6,7,8]$ and method, which uses the fuzzy control system to determine the hysteresis value [9].

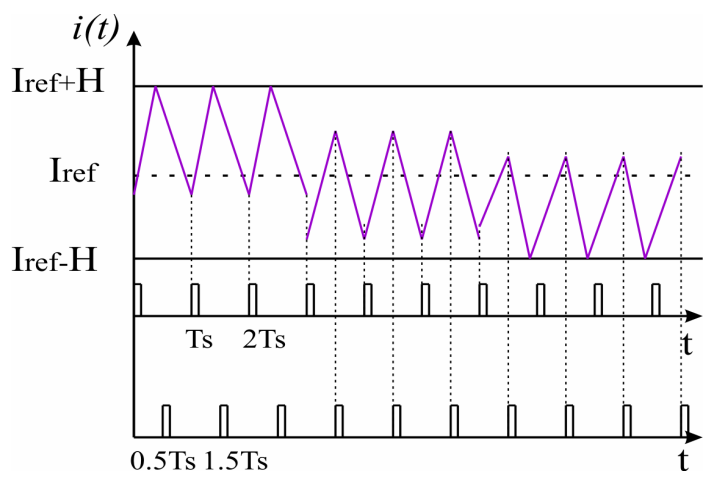

Fig. 2 - Time diagram of hysteresis controlled current regulator with clocked commutation

The time diagrams of relay current regulator with hysteresis adaptation, which explain the essence of the geometrical hysteresis calculation approach, are shown in Fig. 3, where $H$ and $H^{*}$ are real and desirable hysteresises, $\alpha$ and $\beta$ are current curve slope angles, $t_{e r}$ is a time error of real current curve and current reference $I_{r e f}$ intersection (if the real switching frequency is equal to the desirable frequency and the static control error is equal zero, than $t_{e r}=0$ [6]). The hysteresis is calculated by $t_{e r}$ or by the angles $\alpha, \beta$ and current value, depending on accepted simplifications. Thus, the desirable switching frequency $1 / T_{S}$ and current reference $I_{\text {ref }}$ are initial data. The current curve linearization is a main simplification in the geometrical hysteresis calculation method (Fig. 3). But in some practical cases the current curves have nonlinear form [10], and it can lead to mistake in a hysteresis calculation. In this case increasing of the switching frequency can appear when current curve linearization is used (Fig. 4). That's why, an increase of the switching frequency is necessary in order to make a current curve more linear. But computing power of controller, which calculate hysteresis, and increase of losses in switching elements limit a possible switching frequency.

The hysteresis value in the actual period is calculated with data of the previous period (Fig. 3). That's why the hysteresis value is incorrect if a reference current has a big change within one period. In this case the amplitude and frequency of the current reference reduce the output signal accuracy (when the amplitude or frequency grows up). The situation when one can't neglect the reference current changes is shown in Fig. 5. Here we can see, that a desirable frequency can't be obtained by hysteresis adaptation.

A current "lagging" appears and grows with the reference current frequency increase because of limited computing power.

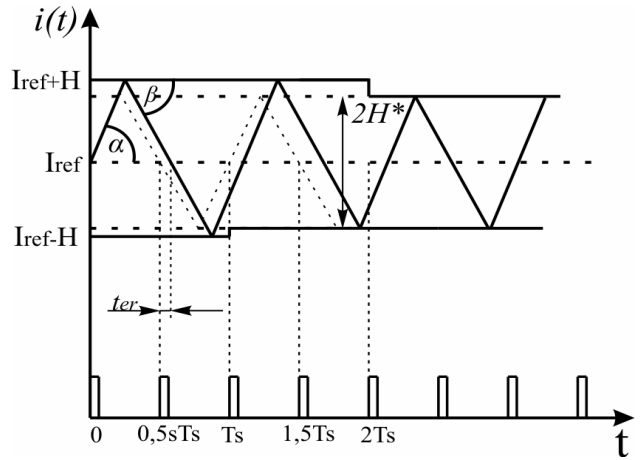

Fig. 3 - Time diagram of the relay current regulator with adaptation of hysteresis by a geometrical method

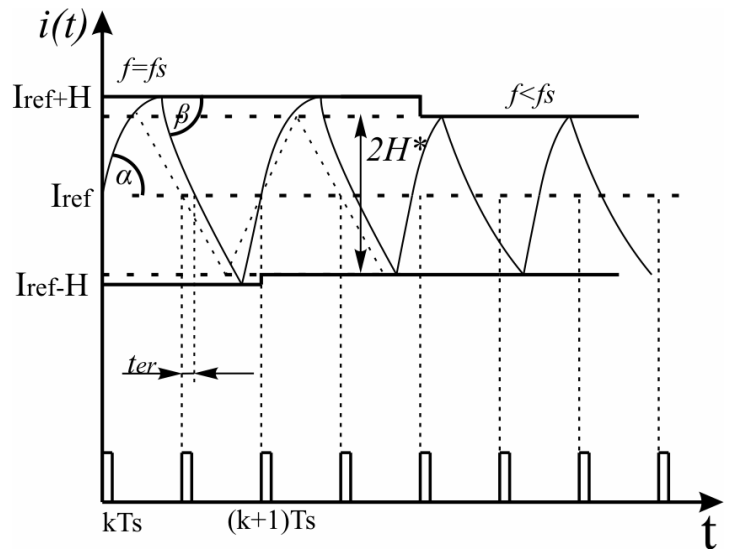

Fig. 4 - Time diagram of relay current regulator with adaptation of hysteresis by geometrical method with nonlinear current curve

The approach of [8] tries to obtain more accuracy of the hysteresis calculation and therefore increases the regulator complication (it needs to know, in the induction motor case, a winding inductance, a source voltage, a flux).

The fuzzy adaptation of the hysteresis is another direction of the hysteresis adaptation method [9]. It doesn't give a good result - static control error are 0.3 A and 0.33 A for "conventional" hysteresis regulator and regulator with fuzzy adaptation of hysteresis correspondingly [9]. But the information about difference between the real and desirable frequency is necessary for an implementation of the relay current regulator with fuzzy adaptation of hysteresis.

The analysis of hysteresis adaptation methods shown, that the hysteresis adaptation algorithms deprive the relay current regulators the main advantages - simplicity of implementation and high quick-action.

The essence of the current reference adaptation method consists in changing of the current reference by the value of static control error [1]. The current reference adaptation implementation consists in including an integrator in the HCCRCC (Fig. 6) [1]. 


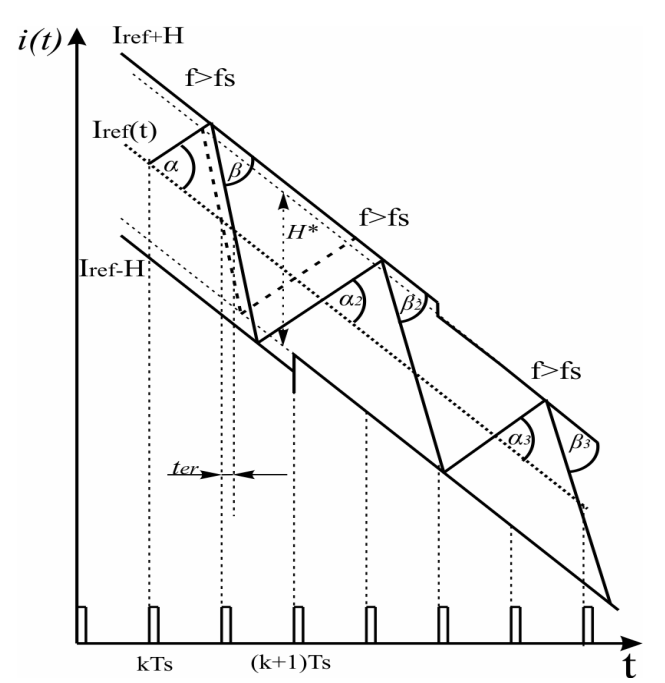

Fig. 5 - Time diagram of relay current regulator with adaptation of hysteresis by geometrical method with sine wave current reference

The HCCRCC with current reference adaptation eliminates the static control error, maintains the permanent switching frequency, can be implemented without controller and doesn't lose quick-action in comparison with the HCCRCC [1]. But it can be provided if the current reference is a constant magnitude only. An appearance of the static control error, when reference current is a variable quantity, is a disadvantage of the HCCRCC with current reference adaptation (hereinafter the sine wave reference current will be considered). This happens at the duty cycle point $d=0.5$. Changing from the algorithm with top-stand relay border to the algorithm with low-stand relay border implements here. Accordingly the control error $e$ changes sign from minus to plus and the integrator output value changes its polarity (the integrator from the current reference adaptation circuit - Fig. 6). Some time need to change the polarity mark of the integrator output value because of inertia, which is determined by the integrator time constant (it is chosen from stability demand [1]). And adaptation of a reference current doesn't work until this process won't complete. At the duty cycle point $d=0.5$ the "degenerative" periodic process appears, when the switching occurs by both synchronization clock sequences [1] (Fig. 3). The current diagram is shown in Fig. 7, where the reference current frequency and the amplitude are equal $50 \mathrm{~Hz}$ and $45 \mathrm{~A}$ correspondently, the source voltage is equal $12.6 \mathrm{~V}$, the load inductance and resistance are equal 117.3

$\mu \mathrm{H}$ and $0.03 \mathrm{Om}$ correspondently, the synchronization period is equal $0.0002 \mathrm{~s}$, the region within the duty cycle $d=0.5$ is marked by ovals. Elimination of HCCRCC with current reference adaptation disadvantage can be obtained by reducing of the hysteresis value, which is determined by a pulsation of current and synchronization frequency. If it is less than the minimal value than bifurcations phenomena can appear in the system [1]. That's why, the calculation method, which can reduce hysteresis value to the minimal quantity in the region of duty cycle $d=0.5$, is necessary to design.

Analysis has shown, that the hysteresis adaptation algorithms deprive the relay current regulator of the basic advantage - simplicity, and demand significant computing expenses. Another disadvantage, that accuracy loss takes place if linearization of current curves and assumption about minor alteration of reference current are used (Fig. 4,5). The adaptation of current reference in HCCRCC eliminates the static control error within the whole range of duty cycle variation (except for the area $d=0.5$ when reference current is a variable quantity), maintains the switching frequency equal to the synchronization frequency and saves the basic advantages of this regulator - simplicity of implementation.

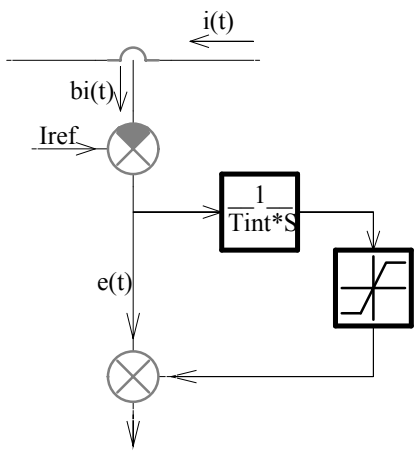

Fig. 6 - Scheme of current reference correction based on an integrator

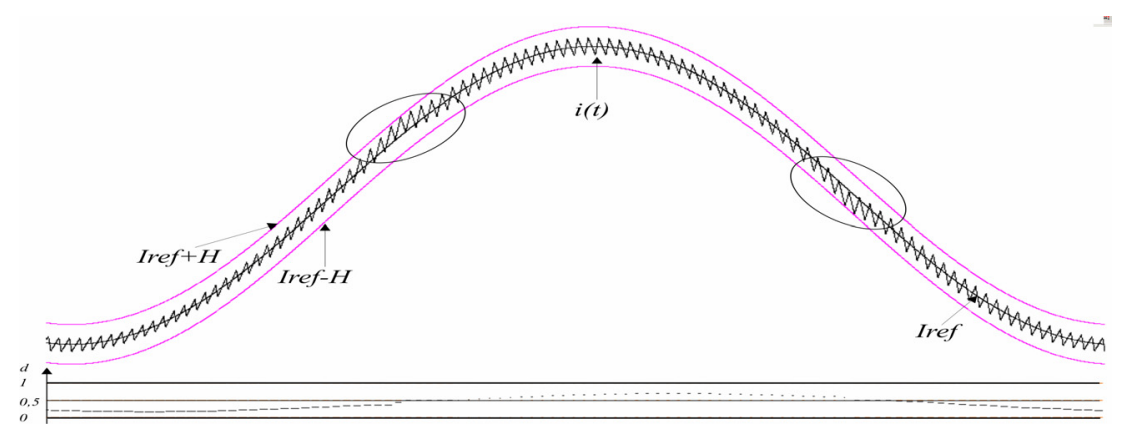

Fig. 7 - Current diagram of HCCRCC with current reference adaptation and sine wave reference current 
Accordingly, it is possible to consider current reference adaptation directions as one of the most perspective.Predicative algorithm of hysteresis calculation, which eliminates undesirable consequence of the integrator using, in the branch of the current reference adaptation (appearance of the static control error in the area $d=0.5$, Fig. 7), and saves the simplicity of HCCRCC, is proposed in this paper.

\section{HYSTERESIS CALCULATION METHOD FOR HYSTERESIS CONTROL CURRENT REGULATOR WITH CLOCKED COMMUTATION AND CURRENT REFERENCE ADAPTATION}

The analysis of HCCRCC with current reference adaptation have shown, that the static control error can to appear at the duty cycle $d=0.5$ if current reference is a variable quantity, as the result of a "degenerative" periodic process [1]. In this case, the determination of the hysteresis value at the duty cycle $\mathrm{d}=0.5$, which being based on the current pulsations, is a purpose of hysteresis calculation method. This method must reduce the static control error and maintain the switching frequency equal to the synchronization frequency.

The method of a hysteresis calculation is shown in Fig. 8, where Irefad is an adapted current reference. Values $\mathrm{H}_{1}$ and $\mathrm{H}_{2}$ are determined as a difference between the current value in the commutation time and the current reference in the same time. Than $\mathrm{H}_{\min }$ is chosen as a maximum value between $\mathrm{H}_{1}$ and $\mathrm{H}_{2}\left(\mathrm{H}_{\min }=\max \left\{\mathrm{H}_{1}, \mathrm{H}_{2}\right\}\right)$, where $\mathrm{H}_{1}$ and $\mathrm{H}_{2}$ must be calculated in the same period $T_{s}$. The $\mathrm{H}_{\min }$ is a hysteresis value in the current synchronization period.

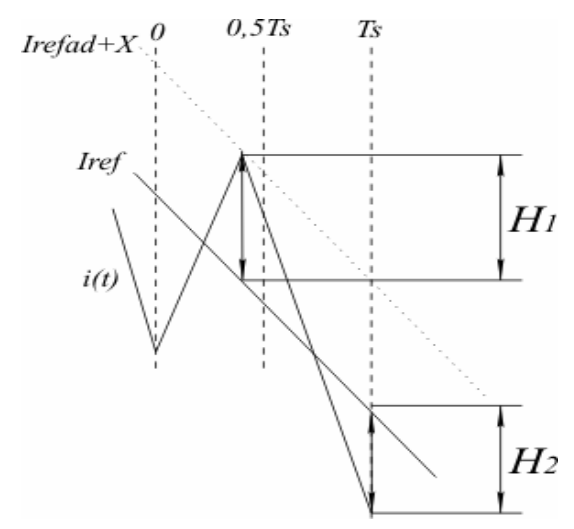

Fig. 8 - Calculation of hysteresis value

An assumption that the current reference changes considerably must be a hysteresis calculation method base. It allows to eliminate the control error that was shown in the example of Fig. 5. In this case, to determinate the hysteresis value at the duty cycle $d=0.5$, the hysteresis calculation method must make a correction at the $H_{m i n}$. This correction can be calculated by means of the current pulse function using:

$$
\Delta i(d)=\frac{V_{g} \cdot T_{S}}{2 \cdot L} d \cdot(1-d) .
$$

The function (1) determines the current pulsation value depending on a duty cycle. It obtains the maximum when $d=0.5$, consequently the $H_{\min }$ value have the following multiplicative correction

$$
M=\frac{d_{0.5} \cdot\left(1-d_{0.5}\right)}{d \cdot(1-d)}=\frac{0.25}{d \cdot(1-d)}=\frac{1}{4 \cdot d \cdot(1-d)} .
$$

The hysteresis value at the duty cycle $d=0.5$ is determined by expression

$$
H_{d=0.5}=M \cdot H_{\min },
$$

where $H_{d=0.5}$ is the hysteresis value that needs in at the duty cycle $d=0.5$.

As one can see from the expressions (1-3) only the current value at a commutation moment and duty cycle value is necessary for hysteresis calculation,. Consequently, the control error, which arises when parameters of system during a work differ from initial values (a winding inductance, a source voltage etc.), eliminates. Also the control error of current curves linearization (Fig. 4) is removed by this method.

\section{HYSTERESIS CALCULATION ALGORITHM FOR HYSTERESIS CONTROL CURRENT REGULATOR WITH CLOCKED COMMUTATION AND CURRENT REFERENCE ADAPTATION}

The hysteresis calculation algorithm, which realizes the method of chapter 2, is shown in Fig. 9, where $X$ is a real value of hysteresis, $t$ is a real time, $k=1,2,3 \ldots$

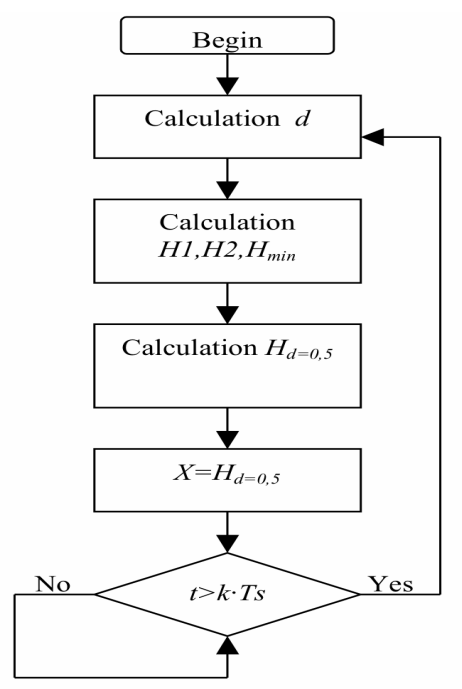

Fig. 9 - Hysteresis calculation algorithm for hysteresis control current regulator with clocked commutation and current reference adaptation 


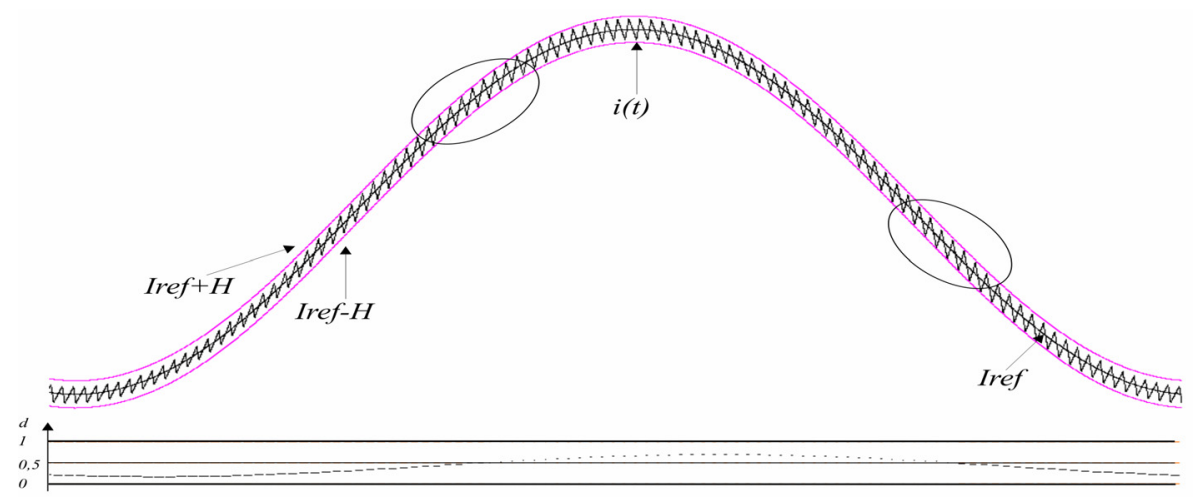

Fig. 10 - Current diagram of HCCRCC with adaptation of current reference and hysteresis

The current diagram of the HCCRCC with adaptation of current reference and hysteresis is shown in Fig. 10. The model parameter values are the same for the diagram of Fig. 7.

Research of the HCCRCC with current reference and hysteresis adaptation shows, that the static control error is less than $0.7 \%$ in the whole range of duty cycle variation (for comparison, HCCRCC with current reference adaptation has the static error more than $5 \%$ for the same current reference). Thus the hysteresis calculation algorithm is simple and can be realized in a real time mode.

\section{CONCLUSION}

The new hysteresis calculation algorithm together with known reference adaptation mechanism was represented in this paper. It allows to control the output sine wave signal amplitude and frequency without loss of quality which appears as a result of current curves linearization. This algorithm is less sensitive to the change of system parameters, because it doesn't use internal parameters of a system for the hysteresis calculation. In difference to the known hysteresis algorithms the consider one plays an auxiliary role and works only at the duty cycle $d=0.5$, and the "simple" HCCRCC provide a regulation quality in the other range of duty cycle variation. Consequently it allows to simplify the calculation algorithm and to make it less demanding to computational power of a controller at implementation.

\section{REFERENCES}

[1] Yu.V. Kolokolov, S.L. Koschinsky, "Dynamics and adaptation of hysteresis controlled current regulator with clocked commutation," Elektrichestvo №6, 2004, pp. 33-43. (Russian)

[2] A.P. Zaitsev, L.Yu. Veitsman, V.A. Podlyagin, Yu.V. Kolokolov, "Relay automatic control system of traction motor current with constant switching frequency," Electrotechnical industry.
Traction and lifting transport equipment, 2 (35) , 1975. pp.3-5. (Russian)

[3] Yu.V. Kolokolov, L.Yu. Veitsman, Zh.T. Zhusurbaliev et al., "Automatic control system of 2-nd generation of high-speed railway train ER200," Electrotechnical Industry. Leading experience and .scientific and technical achievement in application, 8 ( 8 ) , 1988 pp.3-5 (Russian).

[4] A.V. Anunciada, M.M. Silva, "A new current mode control process and applications," IEEE PESC '89 Cof. Rec., 1989, pp 683-694.

[5] L. Malesani, L. Rossetto, P. Tomasin, Al. Zuccato "Digital adaptive hysteresis current control with clocked commutation and wide operation range" IEEE transactions on industry applications, vol. 32, no. 2, march-april 1996

[6] S. Buso, S. Fasolo, L. Malesani, P. Mattavelli "A Dead-Beat Adaptive Hysteresis Current Control," IEEE transaction on industry applications, vol.36, No. 4, 2000

[7] Tae-Won Chun, Meong-Kyu Choi, "Development of adaptive hysteresis band current control strategy of PWM inverter with constant switching frequency," Applied Power Electronics Conference and Exposition, APEC '96. Conference Proceedings 1996., Eleventh Annual IEEE, p. 194-198, 1996

[8] G.H. Bode, D.G. Holmes, "Load independent hysteresis current control of a three level single phase inverter with constant switching frequency," IEEE, p. 14-19, 2001

[9] C. Cecati, S. Corradi, N. Rotondale, "Digital Adaptive Hysteresis Current Control Based on the Fuzzy Logic," ISIE'97 - Guimarsees, Portugal, 1997, p. 1232-1237

[10] I.Ya. Rankis "Parameter optimization for thyristor pulse control system," - Riga: Zinatne. 1985, 186 p. (Russian) 


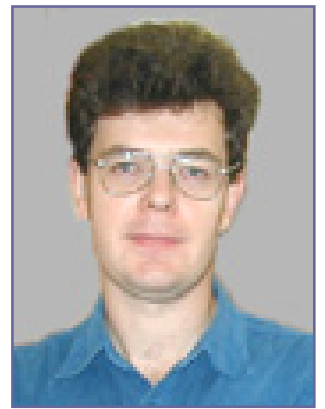

Stanislav L. Koschinsky was born in Orel, Russia on July 31, 1972.

He received the engineer and Ph.D. degrees from Orel State Technical University in 1994 and 1998, respectively. He is presently an Associate Professor in the Department of Electrical and Computer Engineering at the Orel State Technical University. His interests include electromechanical systems dynamics and power electronics.

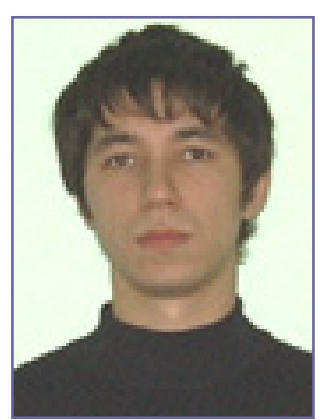

Dmitry O. Tey was born in Orel, Russia, on January 6, 1982. He graduated with honors "Design and Technology of Computer Systems" in 2004 from the Orel State Technical University (Ore/STU), Russia, where he is currently working toward the Ph.D. degree in automation and control of technological processes and productions (in industry).

His main research interests include dc/ac converters, digital control, and robust control of power converters. 